\title{
A PRELIMINARY NOTE ON THE USE OF RETRO- BULBAR PROCTOCAINE ANAESTHESIA FOR THE RELIEF OF INTRACTABLE OCULAR PAIN
}

BY

\author{
L. H, SAVIN and T. M. TYRRELL \\ LONDON
}

RELIEF from prolonged and severe pain in an eye retaining useful vision is often difficult. Ophthalmic pain can sometimes be unbearable, especially in elderly patients. Measures such as the application of heat, the use of leeches, and sedatives are often ineffective. Anaesthesia by the retrobulbar injection of procaine solution though effective is transitory.

Since January, 1940, we have been experimenting in intractable cases with the retrobulbar injection of " proctocaine," and have acquired enough data for a preliminary report on what promises to be a valuable therapeutic procedure. " Proctocaine", is an oily solution of local anaesthetic described by Morgan (1935), and employed by him for the production by injection of anaesthesia of some weeks duration in cases of anal fissure. The solution consists of procaine 1.5 per cent. (for immediate anaesthesia), butyl p. amino-benzoate 6 per cent. (for prolonged anaesthesia after the effect of the procaine has worn off), butyl alcohol 5 per cent. (slightly anaesthetic and to assist solution) in almond oil.

Injected behind painful eyes this solution gives in many cases dramatic and prolonged relief, allowing the patient respite from pain while the eye itself can be treated by ordinary therapeutic measures. The injection is given by the ordinary technique of retrobulbar injection (see de Saint Martin, 1939). Following the makers' advice a No. 8/10 (No. 21.B.W.G., 13") needle is employed with an ordinary 2 c.c. record syringe. Using all aseptic precautions the lower and outer angle of the orbit is palpated. The needle is passed along the outer orbital wall towards the apex for a distance of one inch. About 1.0 to $1.5 \mathrm{c} . \mathrm{c}$. of proctocaine are injected slowly inside the muscle cone in the neighbourhood of the ciliary ganglion. The proctocaine should be warmed to 45 degrees C. Immediate relief from pain should follow the injection and should last up to three weeks.

These injections were first tried by one of us (L. H. S) at Horton Hospital, where the confusions of the first few months of war had forced him to undertake general surgery. After treating anal fissures successfully with proctocaine it was a natural step to use the solution for ophthalmic purposes.

Our final conclusions will be discussed after describing our respective series of cases. 


\section{Cases reported by L.H.S.}

(a) An elderly woman with severe intractable pain, lacrimation, and photophobia from severe bilateral rosacea keratitis. After the injection of 1 c.c. proctocaine into each orbit she could open her eyes and lacrimation and photophobia were much lessened. Partial anaesthesia lasted 28 days during which time the eyes improved under ordinary treatment.

(b) An old woman with severe marginal sclerosing keratitis in both eyes. When first seen she was so depressed as to be almost suicidal. Her pain was completely relieved for 3 weeks by the injection of 1 c.c. proctocaine into each orbit. During this time the eyes improved under treatment.

(c) An old woman with painful bilateral long-standing iridocyclitis. Pain was much relieved by the proctocaine injections. The patient stopped rubbing her eyes all the time, a habit which had previously retarded her recovery.

(d) A case of syphilitic iritis. The pain was in no way relieved. The patient was moving the head during injection and it was thought the proctocaine went outside the muscle cone.

\section{Cases reported by T.M.T.}

(e) An elderly woman who had been attending out-patients for six weeks with a very intractable dendritic ulcer, which would not heal. Pain was intolerable, and corneal sensibility was slightly impaired. After the injection of 1 c.c. proctocaine the pain remained for 15 minutes and then suddenly vanished, presumably from the gradual percolation of the oil. After three days pain returned slightly, but it was never during the next three weeks so bad that she could not sleep. This had been her main complaint prior to the injection. Corneal sensibility was unaltered. The ulcer gradually lessened in extent under local treatment.

(f) A young woman suffering from a fairly severe attack of iritis was much troubled with insomnia. She was given a retrobulbar injection of 1 c.c. proctocaine, with almost immediate relief. The iritis had been present for 14 days before the injection, but cleared up in the next 4 days. There was no loss of corneal sensibility. I do not think that the proctocaine exerted any direct effect on the clearing, but by making the eye less painful to touch, it enabled a rather diffident patient to apply atropine properly, and thus hastened recovery.

(g) A man aged 55 years, who in 1920 had a perforating injury from a thorn. Secondary cataract formed and there was a strand of lens capsule passing forward from the remains of the lens to 
the site of the perforation in the centre of the cornea. For years the eye was painful and irritable. In February, 1940, the capsular band was divided with temporary relief. Later severe iritis occurred, and atropine did not relieve the pain. In jection of 1 c.c. of proctocaine gave some relief, but there was a latent period of 12 hours. The pain later returned but was far less acute. The iritis quieted under treatment.

(h) A man of thirty years of age with acute iritis had not slept for a week. He was given an injection of proctocaine, and immediately developed diplopia on looking down. I had evidently picked off the inferior rectus or its nerve. This diplopia could be elicited for the next week, but did not inconvenience him in any way, as he had to keep the eye covered on account of treatment. All pain was lost from the time of the injection, and in a week the eye was white with $6 / 5$ vision. There was no loss of corneal sensibility.

(i) A young woman (aged 26 years) attended hospital 14 days after the onset of a severe iritis. She was troubled with insomnia from the pain. After a week's unsuccessful local treatment an injection of 1.5 c.c. of proctocaine was given. The pain disappeared completely within ten minutes and did not return. Diplopia developed immediately and lasted two days. There was slight exophthalmos $(1 \mathrm{~m} . \mathrm{m}$. proptosis as measured with the exophthalmometer), probably from using more proctocaine than was necessary. The iritis subsided in 3 days and has not recurred at the time of writing.

(j) A man of 65 years of age with severe iritis of three weeks' standing. He developed atropine irritation, and the pupil was not kept properly dilated, with resulting increase of pain and loss of sleep. After 1 c.c. of proctocaine he lost his pain in 15 minutes. The iritis cleared up on hyoscine drops in 5 days, though at the time of writing there is still conjunctival redness from hyoscine irritation.

(k) A man of 32 years of age with two days' iritis and many posterior synechiae. Pain was severe. He was given a subconjunctival injection of mydricaine, and 1 c.c. of proctocaine retrobulbarly. The pain went in 5 minutes. In two days the only signs of iritis were a small subconjunctival haemorrhage where he had had the mydricaine, and a ring of pigment on the lens capsule. Otherwise the eye was white.

(l) I have used proctocaine in two cases of herpes ophthalmicus with keratitis. Pain was greatly relieved and I saw no ill effects.

$(m)$ Proctocaine also seemed to help a case of recurrent abrasion but here the pain might have improved spontaneously. 
(n) A man of 55 years of age had a blind painful eye with glaucoma of secondary type. I erroneously supposed it to follow thrombosis of the central vein and I advised enucleation which he refused. As a temporary measure I gave a proctocaine retrobulbar injection, done in the usual way, and as far as I could determine given into the right position within the cone of muscles. The injection was completely unsuccessful. Later he consented to enucleation, and the eye was found to have a small melanoma of the choroid with secondary glaucoma. It was indeed fortunate that the injection had proved a failure in relieving his pain. I do not know why the anaesthesia was unsuccessful.

\section{Comments}

1. Retrobulbar injections of proctocaine were performed for 18 painful eyes. In 11 cases the pain was completely relieved, and partially in 5 cases. There were two failures, one due to a faulty injection, the second unexplained.

2. Corneal sensibility was carefully tested both before and after the injections. Usually the sensitivity was lowered, but never completely absent after the injection. We feel that particular care should be taken where retrobulbar injections are given in cases where the corneal sensitivity is lowered as in cases $(e),(l),(m)$. We have seen no neuroparalytic keratitis after these injections, but the patients should be kept under observation.

3. The two cases of diplopia following proctocaine injection both cleared rapidly. Their occurrence did not surprise us, as a sector of the sphincter ani is often temporarily paralysed when an anal fissure is injected with proctocaine. In both cases the injection was given rather far back inside the muscle cone. Patients with good vision in a painful eye should be warned of the possibility of temporary diplopia before giving the injection. Fortunately an intolerably painful eye has usually reduced vision, so that diplopia would not be noticed in such cases.

4. None of our patients showed any sign of toxic symptoms following injection. Proctologists sometimes use 20-30 c.c. with impunity.

5. Comparatively small amounts of the proctocaine have so far been employed because of doubt as to whether the almond oil would be absorbed from the orbit. In two cases some of the oil came forward subconjunctivally. In one case it disappeared in a few days, in the second it remained on view for three weeks. It is generally supposed that the vegetable oils become emulsified after injection into the body and are gradually absorbed. Pending 
accurate information we have so far deemed it unwise to try a second injection where the first was incompletely successful. Experimental work on the absorption of oil would be helpful.

6. Proctocaine was employed because it was the only preparation readily obtainable on the market for prolonged anaesthesia. The proportions of the ingredients were originally planned for effective rectal and anal anaesthesia only. Modifications of the constituents might quite probably give a better solution for orbital use. Further research would seem indicated.

7. If proctocaine is employed for the relief of pain in a blind eye, all possibility of neoplasm should be excluded (see Case $(n)$ ).

Proctocaine retrobulbar injections can be safely recommended for painful eyes with full corneal sensibility and poor vision. If vision is good in a painful eye, the possibility of producing temporary diplopia by the injection must be remembered. Neuroparalytic keratitis is a theoretical possibility if corneal sensitivity is unduly lowered, but so far this complication has not been met with in practice.

We wish to thank Messrs. A. D. Griffith, G. C. Pritchard, A. C. Reid, and H. M. Symons for their kindly advice and collaboration.

\section{REFERENCES}

Morgan, C. Naunton (1935).-Brit. Med.Jl., Vol. II, pp. 938-942.

SaInT-Martin, R. DE (1939).-Traité d'ophtal., Vol. VII, p. 423.

\section{CONVERGENCE WEAKNESS}

BY

\section{O. GAYER MORgaN}

LONDON

I HAVE been interested in these cases of defective convergence for some time, and one of the things that surprised me was that quite a number of people seemed to have no convergence at all, and yet had no symptoms of eye strain with near work or reading.

Stutterheim's monograph was extremely interesting and made me look out more for the cases with defective convergence and symptoms, but I felt that he laid too much stress on the muscles and too little stress on the brain. His method of training tended to underestimate the value of explanation.

The whole condition is very important because of the number of people who show the condition and the severity of the clinical symptoms which can arise from it. 